\title{
ON TOTALLY GEODESIC BOUNDARIES OF HYPERBOLIC 3-MANIFOLDS
}

\author{
By Michiniko FujII
}

\section{Introduction.}

By a hyperbolic manifold, we will mean a Riemannian manifold with constant sectional curvature -1 . In this paper, we study complete oriented hyperbolic 3-manifolds each of which has a totally geodesic boundary. A totally geodesic boundary of such a 3-manifold becomes a hyperbolic surface.

Let $g$ be an integer greater than or equal to 2 and let $\boldsymbol{M}_{g}$ be the Riemann moduli space consisting of all isometry classes of connected closed hyperbolic surfaces of genus $g$. Let $\boldsymbol{S}_{g}$ be the subset of $\boldsymbol{M}_{g}$ consisting of those hyperbolic surfaces which are boundary components of compact oriented hyperbolic 3manifolds with totally geodesic boundary. As pointed out by Soma, there is an argument making use of a theorem of Brooks [1] which shows that $\boldsymbol{S}_{g}$ is dense in $\boldsymbol{M}_{g}$. However it seems that almost nothing is known about the characterization of elements of $\boldsymbol{S}_{g}$. For example, it is by no means easy to construct elements of $\boldsymbol{S}_{g}$ except for obvious ones.

In this paper, we will obtain certain concrete examples of elements of $\boldsymbol{S}_{g}$ by taking a complete hyperbolic 3-manifold with one torus cusp and totally geodesic boundary and then analyzing the effect of the hyperbolic Dehn surgery on the toral end. More precisely, we consider the following. Namely, take any complete hyperbolic 3-manifold $P$ with one torus cusp and connected totally geodesic boundary $\Sigma_{g}$ of genus $g$. Denote $P(p, q)$ the 3 -manifold obtained by performing Dehn surgery on $P$ of type $(p, q)$ on the toral end, where $(p, q)$ is a coprime pair of integers near $\infty$ in $\boldsymbol{R}^{2} \cup\{\infty\}$. Then, using the results of Thurston and Mostow, we see that $P(p, q)$ admits a complete hyperbolic structure with connected totally geodesic boundary $\Sigma_{g}(p, q)$ of genus $g$. For each $g$, we show that there are infinitely many mutually non-isometric $P(p, q)$ 's by considering the lengths of the adjoining closed short geodesics (see [3]). Now in such situations, it seems that the following phenomenon may quite often happen: if $(p, q)$ is close to $\infty$ in $\boldsymbol{R}^{2} \cup\{\infty\}$, then the original surface $\Sigma_{g}$ and the resulting surface $\Sigma_{g}(p, q)$ are different in the Teichmüller space which are very close to each other, so they are also different in the moduli space $\boldsymbol{M}_{g}$. However at present, there are no general theory about this. Moreover, as far as the author

Received November 25, 1991. 
knows, there has not been known any such hyperbolic 3-manifolds where the above phenomenon has been explicitly confirmed to occur. In this paper, we illustrate the above on a particular example $M$ which we will construct in $\S 1$. This 3-manifold $M$ has a complete hyperbolic structure with one torus cusp and connected totally geodesic boundary $\Sigma$ of genus 2 and is one of the easiest ones to deal with. From this example $M$, we explicitly construct infinitely many mutually non-isometric connected closed hyperbolic surfaces $\Sigma(p, q)$ 's which are elements of $\boldsymbol{S}_{2}$ and converge to $\Sigma$ as $(p, q)$ goes to $\infty$. We mention that Neumann-Reid showed, by contrast, that for each $g$, there is a complete hyperbolic 3-manifold with one torus cusp and totally geodesic boundary of genus $g$ such that deformations induced by hyperbolic Dehn surgery of the cusp leave the boundary surface invariant (see [7]).

We show the above by means of the move of the flat structure of the cusp torus of $D M$ (the double of $M$ along $\partial M$ ) with respect to the hyperbolic Dehn surgery on the other toral end of $D M$. The move is shown in the following way. Namely, first of all, we decompose $D M$ into ideal tetrahedra in $\S 1$. Next we write down the deformation space in our situation in $\S 2$. Finally in $\S 3$, we describe the isomorphism type $\tau$ of the flat structure corresponding to the cusp torus in question in terms of the tetrahedral parameters. Though there are thirty-six parameters and matrices of large size appear, all necessary calculations are worked out just within the field $\boldsymbol{Q}(\sqrt{2}, \sqrt{-1})$. We can thus show successfully that the differentiation of the second order of $\tau$ with respect to the parameters is not zero.

\section{An ideal tetrahedral decomposition of a hyperbolic 3-manifold with two torus cusps.}

In this section, we construct a 3-manifold $M$ which has a complete hyperbolic structure with one torus cusp and connected totally geodesic boundary $\Sigma$ of genus 2 and then concretely find out an ideal tetrahedral decomposition of the double $D M$ of $M$ along $\partial M$ (there exists at least one such a decomposition by [2]).

Let $S$ be an ideal tetrahedron in the hyperbolic 3 -space $\boldsymbol{H}^{3}$. Namely, $S$ is a geodesic tetrahedron with all vertices at infinity $\partial \boldsymbol{H}^{3}$. An ideal tetrahedron is described (up to isometry) by a single complex number $z$ with positive imaginary part such that the Euclidean triangle cut out of any vertex of $S$ by a horosphere section is similar to the triangle with vertices 0,1 and $z$ (see Fig. 1.1(a)). We write $S=S(z)$. The three numbers $z, z^{\prime}:=(z-1) / z$ and $z^{\prime \prime}:=1 /(1-z)$ give the same tetrahedron: to specify $z$ uniquely, we must pick an edge of $S$ (the dihedral angle at this edge will be $\arg (z)$ ). Then to each edge of $S$ is associated one of the three numbers $z, z^{\prime}$ and $z^{\prime \prime}$, the modulus of the edge, opposite edges of $S$ having the same modulus (see Fig. 1.1(b)). 


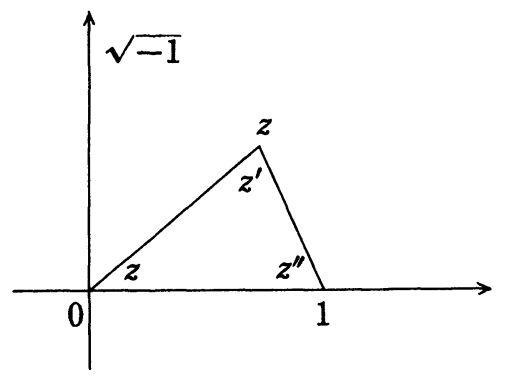

Fig. 1.1(a)

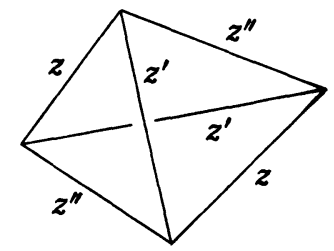

Fig. 1.1(b)

Let us consider a geodesic polyhedron in $\boldsymbol{H}^{3}$ as indicated in Fig. 1.2 and identify the faces as follows: glue $A_{1}$ to $C_{1}, B_{1}$ to $C_{2}, D_{1}$ to $A_{2}, E_{1}$ to $H_{2}, F_{1}$ to $H_{1}, G_{1}$ to $F_{2}, B_{2}$ to $D_{2}$ and $E_{2}$ to $G_{2}$ respectively. Then we obtain the complete hyperbolic 3-manifold $M$ with one torus cusp and connected totally geodesic boundary $\Sigma$ of genus 2 .

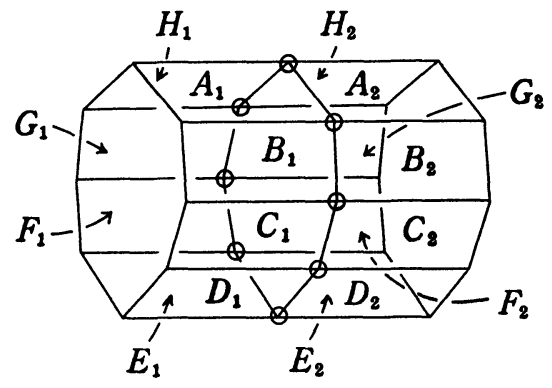

Fig. 1.2. The vertices $\bigcirc$ are ideal ones. All boundary faces of this polyhedron are all totally geodesic. All dihedral angles are $\pi / 2$. Right-angled sixteen quadrilaterals with two ideal vertices are isometric to each other. The totally geodesic boundary $\Sigma$ of $M$ consists of the two right-angled octagons.

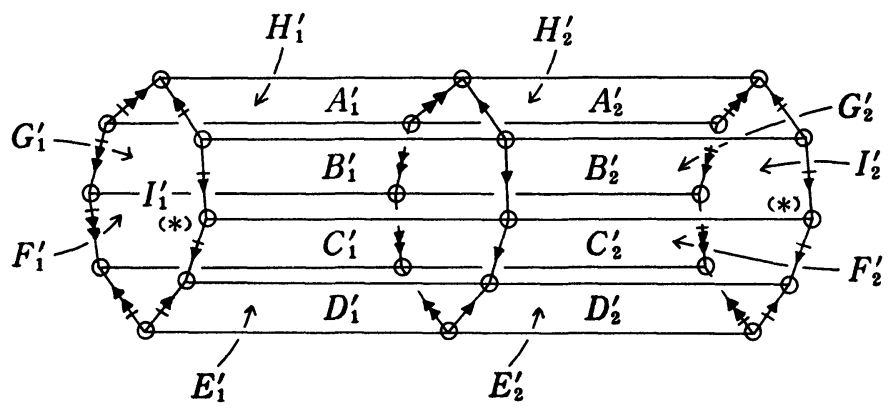

Fig. 1.3. Glue $A_{1}^{\prime}$ to $C_{1}^{\prime}, B_{1}^{\prime}$ to $C_{2}^{\prime}, D_{1}^{\prime}$ to $A_{2}^{\prime}, E_{1}^{\prime}$ to $H_{2}^{\prime}, F_{1}^{\prime}$, to $H_{1}^{\prime} G_{1}^{\prime}$ to $F_{2}^{\prime}, B_{2}^{\prime}$ to $D_{2}^{\prime}, E_{2}^{\prime}$ to $G_{2}^{\prime}$ and $I_{1}^{\prime}$ to $I_{2}^{\prime}$ respectively so that the arrows and the stars (*) on the faces $I_{1}^{\prime}$ and $I_{2}^{\prime}$ are identified with each other. 
The fundamental domain of the double $D M$ of $M$ along $\partial M$ in the hyperbolic 3 -space $\boldsymbol{H}^{3}$ is an ideal polyhedron as illustrated in Fig. 1.3 and $D M$ is obtained by identifying the faces of this ideal polyhedron according to the gluing diagram.

Now subdivide this polyhedron into thirty-six ideal tetrahedra $S_{1}:=S\left(z_{1}\right), \cdots$, $S_{36}:=S\left(z_{36}\right)$ as in Fig. 1.4. We have obtained an ideal triangulation of $D M$ by thirty-six simplices:

$$
D M=S_{1} \cup \cdots \cup S_{36}
$$
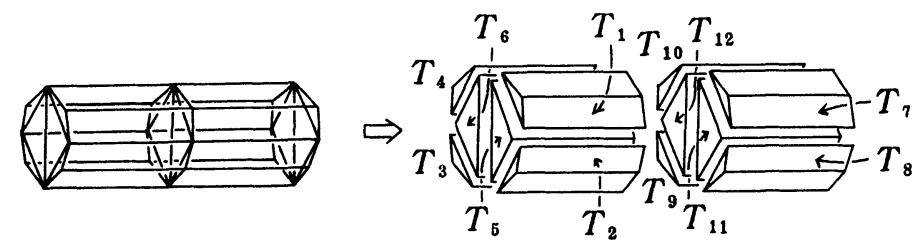

Fig. 1.4(a). Decompose the polyhedron into the twelve triangular prisms $T_{1}, \cdots, T_{12}$

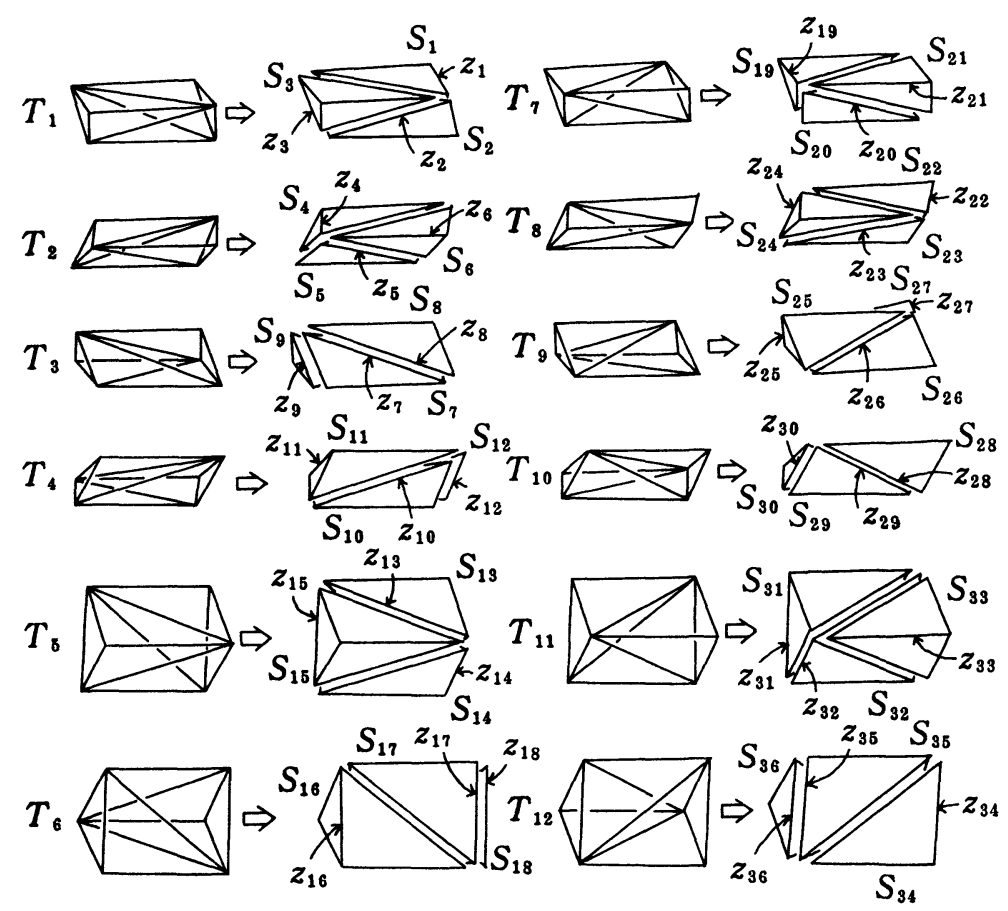

Fig. 1.4(b). Decompose each triangular prism into three tetrahedra $S_{\text {, }}$. 


\section{A deformation space of the hyperbolic structure on the 3-manifold $D M$.}

In this section we treat deformations of the hyperbolic structure on the 3manifold $D M$. Especially we will consider deformations leaving one of the two toral ends of $D M$ complete (i.e. on these deformations one of the two ends remains cuspidal). Let $\mathcal{E}_{1}, \mathcal{E}_{2}$ be the two toral ends of $D M$. The argument in this section is given in [8] under the generalized situation.

Let $z_{t}^{o}$ be as follows:

$$
\begin{aligned}
& z_{1}^{o}:=z_{11}^{o}:=z_{17}^{o}:=z_{22}^{o}:=z_{26}^{o}:=z_{32}^{o}:=(2+\sqrt{2})(1+\sqrt{-1}) / 2 \\
& z_{2}^{o}:=z_{5}^{o}:=z_{7}^{o}:=z_{10}^{o}:=z_{20}^{o}:=z_{23}^{o}:=z_{25}^{o}:=z_{28}^{o}:=-\sqrt{2}(1-\sqrt{-1}) / 2 \\
& z_{3}^{o}:=z_{6}^{o}:=z_{9}^{o}:=z_{12}^{o}:=z_{21}^{o}:=z_{24}^{o}:=z_{27}^{o}:=z_{30}^{o}:=\sqrt{-1} \\
& z_{4}^{o}:=z_{8}^{o}:=z_{14}^{o}:=z_{19}^{o}:=z_{29}^{o}:=z_{35}^{o}:=(2-\sqrt{2})(1+\sqrt{-1}) / 2 \\
& z_{13}^{o}:=z_{34}^{o}:=(-1+\sqrt{2}) \sqrt{-1} \\
& z_{15}^{o}:=z_{18}^{o}:=z_{33}^{o}:=z_{36}^{o}:=\sqrt{2}(1+\sqrt{-1}) / 2 \\
& z_{16}^{o}:=z_{31}^{o}:=(1+\sqrt{2}) \sqrt{-1} .
\end{aligned}
$$

Let us call the point $z^{o}:=\left(z_{1}^{o}, \cdots, z_{36}^{o}\right) \in C^{36}$ the original solution. This point $z^{o}$ gives the same state as in Fig. 1.4, then $D M$ is the complete hyperbolic 3manifold with two torus cusps when $z=z^{0}$, where $z=\left(z_{1}, \cdots, z_{36}\right)$. In the case where $\boldsymbol{z}=\boldsymbol{z}^{0}$, the link $L_{\imath}$ of the ideal vertex $v_{i}$ which corresponds to the toral end $\mathcal{E}_{\imath}$ has a Euclidean triangulation induced by the ideal tetrahedral decomposition as shown in Fig. 2.1(a) and (b) for each $i(i=1,2)$, where we have included labels for the tetrahedral parameters $z_{1}, \cdots, z_{36}$ of the thirty-six tetrahedra.

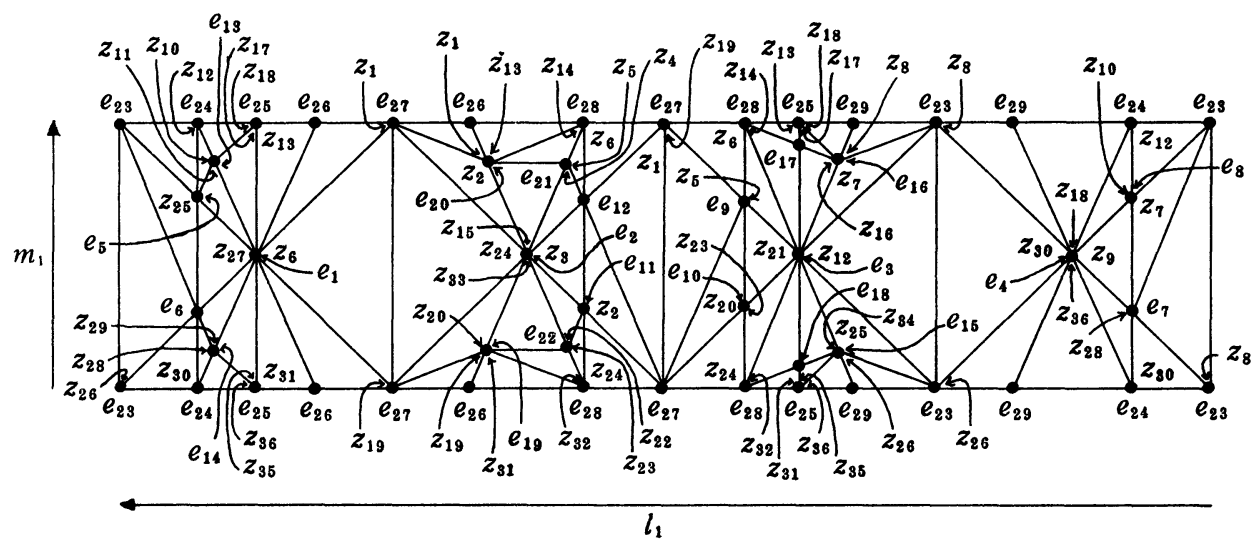

Fig. 2.1(a) 


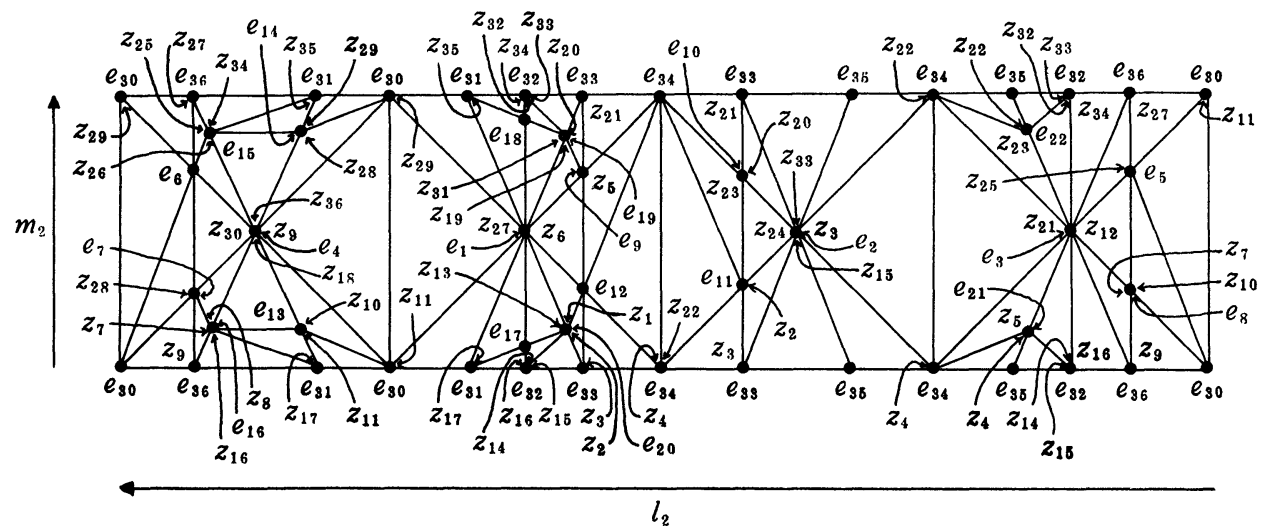

Fig. 2.1(b). The Euclidean triangulation corresponding to the link $L_{2}$. We omitted the labels $z_{\jmath}^{\prime}, z_{\jmath}^{\prime \prime}(\jmath=1, \cdots, 36)$ to simplify the picture.

Even if $\boldsymbol{z} \neq \boldsymbol{z}^{0}$, the triangulation has the same combinatorial type as in the case where $z=z^{\circ}$.

Choose generators $m_{\imath}, l_{\imath}$ for $\pi_{1}\left(L_{\imath}\right)$ as indicated in Fig. 2.1(a) and (b) for each $\imath(\imath=1,2)$.

We can read off from Fig. 2.1(a) and (b) the consistency relation $h_{k}=1$ at the edge $e_{k}$ for each $k(k=1, \cdots, 36)$ :

$$
\begin{aligned}
& h_{1}:=z_{1}^{\prime} z_{6} z_{11}^{\prime} z_{13}^{\prime \prime} z_{17}^{\prime} z_{19}^{\prime \prime} z_{27} z_{29}^{\prime \prime} z_{31}^{\prime} z_{35}^{\prime \prime}=1, \\
& h_{2}:=z_{2}^{\prime} z_{3} z_{4}^{\prime \prime} z_{15} z_{20}^{\prime \prime} z_{22}^{\prime} z_{24} z_{33}=1, \\
& h_{3}:=z_{5}^{\prime \prime} z_{7}^{\prime \prime} z_{12} z_{14}^{\prime \prime} z_{16}^{\prime} z_{21} z_{23}^{\prime} z_{25}^{\prime} z_{32}^{\prime} z_{34}^{\prime \prime}=1, \\
& h_{4}:=z_{8}^{\prime \prime} z_{9} z_{10}^{\prime} z_{18} z_{26}^{\prime} z_{28}^{\prime \prime} z_{30} z_{36}=1, \\
& h_{5}:=z_{10}^{\prime} z_{11}^{\prime \prime} z_{12}^{\prime} z_{25} z_{27}^{\prime \prime}=1, \\
& h_{6}:=z_{25}^{\prime} z_{26}^{\prime \prime} z_{27}^{\prime} z_{28}^{\prime \prime} z_{29}^{\prime} z_{30}^{\prime \prime}=1, \\
& h_{7}:=z_{7}^{\prime \prime} z_{8}^{\prime} z_{9}^{\prime \prime} z_{28} z_{30}^{\prime}=1, \\
& h_{8}:=z_{7} z_{9}^{\prime} z_{10} z_{12}^{\prime \prime}=1, \\
& h_{9}:=z_{5} z_{6}^{\prime} z_{19}^{\prime} z_{20}^{\prime \prime} z_{21}^{\prime \prime}=1, \\
& h_{10}:=z_{20} z_{21}^{\prime} z_{23} z_{21}^{\prime \prime}=1, \\
& h_{11}:=z_{2} z_{3}^{\prime \prime} z_{22}^{\prime \prime} z_{23}^{\prime} z_{24}^{\prime}=1, \\
& h_{12}:=z_{1}^{\prime \prime} z_{2}^{\prime} z_{3}^{\prime} z_{4}^{\prime} z_{5}^{\prime \prime} z_{6}^{\prime \prime}=1, \\
& h_{13}:=z_{10} z_{11} z_{17}^{\prime \prime} z_{18}^{\prime}=1, \\
& h_{14}:=z_{28} z_{29} z_{35}^{\prime} z_{36}^{\prime \prime}=1, \\
& h_{15}:=z_{25} z_{26} z_{34} z_{35}^{\prime \prime} z_{36}^{\prime}=1,
\end{aligned}
$$




$$
\begin{aligned}
& h_{16}:=z_{7} z_{8} z_{16} z_{17}^{\prime} z_{18}^{\prime \prime}=1, \\
& h_{17}:=z_{13}^{\prime} z_{14}^{\prime} z_{16}^{\prime \prime} z_{17}^{\prime \prime}=1, \\
& h_{18}:=z_{31}^{\prime \prime} z_{32}^{\prime \prime} z_{34}^{\prime} z_{35}^{\prime}=1, \\
& h_{19}:=z_{19} z_{20} z_{31} z_{32}^{\prime} z_{33}^{\prime \prime}=1, \\
& h_{20}:=z_{1} z_{2} z_{13} z_{14}^{\prime \prime} z_{15}^{\prime}=1, \\
& h_{21}:=z_{4} z_{5} z_{14}^{\prime} z_{15}^{\prime \prime}=1, \\
& h_{22}:=z_{22} z_{23} z_{32}^{\prime \prime} z_{33}^{\prime}=1, \\
& h_{23}:=z_{7}^{\prime} z_{8} z_{8}^{\prime \prime} z_{12}^{\prime} z_{12}^{\prime \prime} z_{25}^{\prime \prime} z_{26} z_{26}^{\prime} z_{30}^{\prime} z_{30}^{\prime \prime}=1, \\
& h_{24}:=z_{10}^{\prime \prime} z_{12} z_{18}^{\prime \prime} z_{28}^{\prime} z_{30} z_{36}^{\prime}=1, \\
& h_{25}:=z_{13} z_{17} z_{18} z_{31} z_{35} z_{36}=1, \\
& h_{26}:=z_{1}^{\prime \prime} z_{13}^{\prime} z_{19}^{\prime} z_{31}^{\prime \prime}=1, \\
& h_{27}:=z_{1} z_{1}^{\prime} z_{2}^{\prime \prime} z_{6}^{\prime} z_{6}^{\prime \prime} z_{19} z_{19}^{\prime \prime} z_{20}^{\prime} z_{24}^{\prime} z_{24}^{\prime \prime}=1, \\
& h_{28}:=z_{5}^{\prime} z_{6} z_{13}^{\prime \prime} z_{14} z_{23}^{\prime \prime} z_{24} z_{31}^{\prime} z_{32}=1, \\
& h_{29}:=z_{8}^{\prime} z_{18}^{\prime} z_{26}^{\prime \prime} z_{36}^{\prime \prime}=1, \\
& h_{30}:=z_{9}^{\prime} z_{9}^{\prime \prime} z_{10}^{\prime \prime} z_{11} z_{11}^{\prime} z_{27}^{\prime} z_{27}^{\prime \prime} z_{28}^{\prime} z_{29} z_{29}^{\prime \prime}=1, \\
& h_{31}:=z_{11}^{\prime \prime} z_{16}^{\prime} z_{17} z_{29}^{\prime} z_{34}^{\prime \prime} z_{35}=1, \\
& h_{32}:=z_{14} z_{15} z_{16} z_{32} z_{33} z_{34}=1, \\
& h_{33}:=z_{2}^{\prime \prime} z_{3} z_{15}^{\prime \prime} z_{20}^{\prime} z_{21} z_{33}^{\prime}=1, \\
& h_{34}:=z_{3}^{\prime} z_{3}^{\prime \prime} z_{4} z_{4}^{\prime \prime} z_{5}^{\prime} z_{21}^{\prime} z_{21}^{\prime \prime} z_{22} z_{22}^{\prime} z_{23}^{\prime \prime}=1, \\
& h_{35}:=z_{4}^{\prime} z_{15}^{\prime} z_{22}^{\prime \prime} z_{33}^{\prime \prime}=1, \\
& h_{36}:=z_{7}^{\prime} z_{9} z_{16}^{\prime \prime} z_{25}^{\prime \prime} z_{27} z_{34}^{\prime}=1 . \\
& n_{3}
\end{aligned}
$$

Since $z_{t}^{\prime}=\left(z_{t}-1\right) / z_{t}$ and $z_{t}^{\prime \prime}=1 /\left(1-z_{\imath}\right)$, we have the relations $h_{1} \cdots h_{36}=1$ and $h_{23} \cdots h_{29}=h_{30} \cdots h_{36}$. Then these thirty-six relations $h_{1}=1, \cdots, h_{36}=1$ are simplified to the thirty-four relations:

$$
g_{\jmath}:= \begin{cases}h_{\jmath+1}=1 & \jmath=1, \cdots, 26 \\ h_{\jmath+2}=1 & j=27, \cdots, 34\end{cases}
$$

Similarly, we can read off the parameters $u_{\imath}, v_{i}$ which describe the holonomy of the $\left(\boldsymbol{C}^{\times}, \boldsymbol{C} \backslash\{0\}\right)$-structure of the meridian $m_{\imath}$ and the longitude $l_{\imath}$ at the cusp torus corresponding to the link $L_{\imath}$ for each $i(i=1,2)$, where $C^{\times}$is the multiplicative group of non-zero complex numbers. In particular, the parameter $u_{1}$ is represented as follows:

$$
u_{1}=-z_{30}^{\prime} z_{26} z_{26}^{\prime} z_{25}^{\prime \prime} z_{12}^{\prime \prime} \text {. }
$$


The pair $\left(u_{1}, u_{2}\right)$ can be taken as the analytic Dehn surgery parameter as discussed in [6] and [8], in which case $z_{1}, \cdots, z_{36}$, constrained by the equations (*), become complex analytic functions of this parameter.

For the complete structure on $D M$ we have

$$
u_{1}=v_{1}=u_{2}=v_{2}=1 \text {. }
$$

The original solution $z^{0}$ satisfies the above equations $\left(^{*}\right)$ and $\left(^{* *}\right)$. In this paper, we only deal with the tetrahedral parameter $\boldsymbol{z}$ near $\boldsymbol{z}^{0}$. Since $\pi_{1}\left(L_{\imath}\right)$ is abelian, $u_{i}=1$ induces $v_{i}=1$.

Now consider the space

$$
W:=\left\{z=\left(z_{1}, \cdots, z_{36}\right) \in C^{36} \mid z \text { is near } z^{o}, z \text { satisfies }\left(^{*}\right) \text { and } u_{1}=1\right\} .
$$

This space $W$ is the deformation space of the hyperbolic structure on $D M$ in which we let the end $\mathcal{E}_{1}$ corresponding to $L_{1}$ remain to be complete.

\section{Deformations of the flat structure of the cusp torus of the hyperbolic 3-manifold $D M$ with respect to the hyperbolic Dehn surgery.}

In this section we consider the move of the flat structure of the cusp torus corresponding to $\mathcal{E}_{1}$ of the hyperbolic 3-manifold $D M$ with respect to the deformations which are included in the deformation space $W$ in $\S 2$.

The real Dehn surgery parameters $\left(p_{1}, q_{1}\right)$ and $\left(p_{2}, q_{2}\right)$ are determined by the equations :

$$
\begin{aligned}
& p_{1} \log u_{1}+q_{1} \log v_{1}=2 \pi \sqrt{-1} \\
& p_{2} \log u_{2}+q_{2} \log v_{2}=2 \pi \sqrt{ }-1
\end{aligned}
$$

(we take $\left(p_{i}, q_{\imath}\right)=\infty$ if $u_{\imath}=1$ for each $i(\imath=1,2)$ ).

Let $D M\left(p_{1}, q_{1} ; p_{2}, q_{2}\right)$ be the hyperbolic 3-manifold obtained by performing the hyperbolic Dehn surgeries of types $\left(p_{1}, q_{1}\right),\left(p_{2}, q_{2}\right)$ on the two framed toral ends of $D M$ respectively. If the ordered pair $\left(p_{\imath}, q_{\imath}\right)$ is replaced by the symbol $\infty$, this means that the torus cusp is left unsurgered. Thus $D M\left(\infty ; p_{2}, q_{2}\right)$ is the hyperbolic 3-manifold obtained by performing the hyperbolic Dehn surgery of type $\left(p_{2}, q_{2}\right)$ on the framed toral end $\mathcal{E}_{2}$ while the toral end $\mathcal{E}_{1}$ remains to be complete.

The isomorphism type of the flat torus corresponding to $\mathcal{E}_{1}$ of $D M\left(\infty ; p_{2}, q_{2}\right)$ as a complex torus $C / \Lambda$ is described by the complex parameter $\tau=\tau_{1} / \tau_{2}$ with positive imaginary part, after choosing an oriented base $\tau_{1}, \tau_{2}$ for the lattice $\Lambda$ (see Fig. 3.1 and Fig. 3.2). By elementary calculation from Fig. 2.1(a) and Fig. 3.1, one can express $\tau$ in terms of $z_{1}, \cdots, z_{36}$ as

$$
\begin{aligned}
\tau & =\tau\left(z_{1}, \cdots, z_{36}\right) \\
& =\sum_{n=1}^{14}(-1)^{n+1} a_{1} a_{2} \cdots a_{n-1} a_{n}
\end{aligned}
$$


where $a_{1}:=z_{8} z_{30}^{\prime \prime}, \quad a_{2}:=z_{30} z_{28}^{\prime} z_{36}^{\prime}, \quad a_{3}:=z_{36}^{\prime \prime} z_{26}^{\prime \prime}, \quad a_{4}:=z_{26} z_{30}^{\prime} z_{12}^{\prime \prime} z_{25}^{\prime \prime} z_{26}^{\prime}, \quad a_{5}:=z_{26}^{\prime \prime} z_{36}^{\prime \prime}, \quad a_{6}:=$ $z_{36} z_{35} z_{31}, a_{7}:=z_{31}^{\prime} z_{32} z_{23}^{\prime \prime} z_{24}, \quad a_{8}:=z_{24}^{\prime} z_{20}^{\prime} z_{19}^{\prime \prime} z_{1}^{\prime} z_{2}^{\prime \prime} z_{24}^{\prime \prime}, a_{9}:=z_{24} z_{23}^{\prime \prime} z_{32} z_{31}^{\prime}, a_{10}:=z_{31}^{\prime \prime} z_{19}^{\prime}, a_{11}:=$ $z_{19}^{\prime \prime} z_{20}^{\prime} z_{24}^{\prime} z_{6}^{\prime \prime} z_{19}, a_{12}:=z_{19}^{\prime} z_{31}^{\prime \prime}, a_{13}:=z_{31} z_{35} z_{36}$ and $a_{14}:=z_{36}^{\prime} z_{28}^{\prime} z_{30}$.

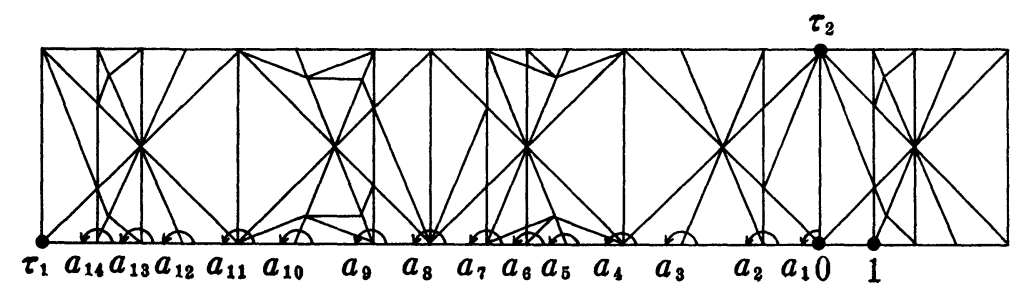

Fig. 3.1. The cusp torus corresponding to $\mathcal{E}_{1}$ developed on complex plane with the oriented base $\tau_{1}, \tau_{2}$ for the lattice $\Lambda$ drawn in.

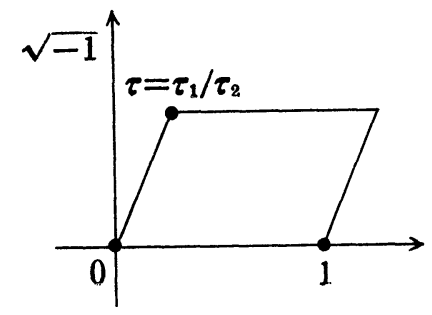

Fig. 3.2. The fundamental domain for the flat torus.

Let $g_{j}(j=1, \cdots, 34)$ be the rational functions as given in $\S 2$, and let $g_{35}=u_{1}$. We express each $g_{\jmath}(\jmath=1, \cdots, 34,35)$ in an irreducible form, that is, $g_{\jmath}=n_{j} / d_{\jmath}$, where $n_{j}$ and $d_{j}$ are polynomials on $z_{1}, \cdots, z_{36}$ without common factors. Then the polynomial $f_{j}:=n_{j}-d$, is determined from $g$, uniquely up to scalar. We can choose such $f_{j}$ 's so that the equations (***) $f_{\jmath}=0(\jmath=1, \cdots, 34,35)$ are given as follows:

$$
\begin{aligned}
f_{1}:= & \left(z_{2}-1\right) z_{3} z_{15}\left(z_{22}-1\right) z_{24} z_{33}-z_{2}\left(z_{4}-1\right)\left(z_{20}-1\right) z_{22}=0, \\
f_{2}:= & z_{12}\left(z_{16}-1\right) z_{21}\left(z_{23}-1\right)\left(z_{25}-1\right)\left(z_{32}-1\right) \\
& \quad-\left(z_{5}-1\right)\left(z_{7}-1\right)\left(z_{14}-1\right) z_{16} z_{23} z_{25} z_{32}\left(z_{34}-1\right)=0, \\
f_{3}:= & z_{9}\left(z_{10}-1\right) z_{18}\left(z_{26}-1\right) z_{30} z_{36}-\left(z_{8}-1\right) z_{10} z_{26}\left(z_{28}-1\right)=0, \\
f_{4}:= & \left(z_{10}-1\right)\left(z_{12}-1\right) z_{25}-z_{10}\left(z_{11}-1\right) z_{12}\left(z_{27}-1\right)=0, \\
f_{5}:= & \left(z_{25}-1\right)\left(z_{27}-1\right)\left(z_{29}-1\right)+z_{25}\left(z_{26}-1\right) z_{27}\left(z_{28}-1\right) z_{29}\left(z_{30}-1\right)=0, \\
f_{6}:= & \left(z_{8}-1\right) z_{28}\left(z_{30}-1\right)-\left(z_{7}-1\right) z_{8}\left(z_{9}-1\right) z_{30}=0, \\
f_{7}:= & z_{7}\left(z_{9}-1\right) z_{10}+z_{9}\left(z_{12}-1\right)=0, \\
f_{8}:= & z_{5}\left(z_{6}-1\right)\left(z_{19}-1\right)-z_{6} z_{19}\left(z_{20}-1\right)\left(z_{21}-1\right)=0, \\
f_{9}:= & z_{20}\left(z_{21}-1\right) z_{23}+z_{21}\left(z_{24}-1\right)=0,
\end{aligned}
$$




$$
\begin{aligned}
& f_{10}:=z_{2}\left(z_{23}-1\right)\left(z_{24}-1\right)-\left(z_{3}-1\right)\left(z_{22}-1\right) z_{23} z_{24}=0 \text {, } \\
& f_{11}:=\left(z_{2}-1\right)\left(z_{3}-1\right)\left(z_{4}-1\right)+\left(z_{1}-1\right) z_{2} z_{3} z_{4}\left(z_{5}-1\right)\left(z_{6}-1\right)=0 \text {, } \\
& f_{12}:=z_{10} z_{11}\left(z_{18}-1\right)+\left(z_{17}-1\right) z_{18}=0 \text {, } \\
& f_{13}:=z_{28} z_{29}\left(z_{35}-1\right)+z_{35}\left(z_{36}-1\right)=0 \text {, } \\
& f_{14}:=z_{25} z_{26} z_{34}\left(z_{36}-1\right)+\left(z_{35}-1\right) z_{36}=0 \text {, } \\
& f_{15}:=z_{7} z_{8} z_{16}\left(z_{17}-1\right)+z_{17}\left(z_{18}-1\right)=0 \text {, } \\
& f_{16}:=\left(z_{13}-1\right)\left(z_{14}-1\right)-z_{13} z_{14}\left(z_{16}-1\right)\left(z_{17}-1\right)=0 \text {, } \\
& f_{17}:=\left(z_{34}-1\right)\left(z_{35}-1\right)-\left(z_{31}-1\right)\left(z_{32}-1\right) z_{34} z_{35}=0 \text {, } \\
& f_{18}:=z_{19} z_{20} z_{31}\left(z_{32}-1\right)+z_{32}\left(z_{33}-1\right)=0 \text {, } \\
& f_{19}:=z_{1} z_{2} z_{13}\left(z_{15}-1\right)+\left(z_{14}-1\right) z_{15}=0 \text {, } \\
& f_{20}:=z_{4} z_{5}\left(z_{14}-1\right)+z_{14}\left(z_{15}-1\right)=0 \text {, } \\
& f_{21}:=z_{22} z_{23}\left(z_{33}-1\right)+\left(z_{32}-1\right) z_{33}=0 \text {, } \\
& f_{22}:=\left(z_{7}-1\right) z_{8}\left(z_{26}-1\right)-z_{7}\left(z_{8}-1\right) z_{12}\left(z_{25}-1\right) z_{30}=0 \text {, } \\
& f_{23}:=z_{12}\left(z_{28}-1\right) z_{30}\left(z_{36}-1\right)-\left(z_{10}-1\right)\left(z_{18}-1\right) z_{28} z_{36}=0 \text {, } \\
& f_{24}:=z_{13} z_{17} z_{18} z_{31} z_{35} z_{36}-1=0 \text {, } \\
& f_{25}:=\left(z_{13}-1\right)\left(z_{19}-1\right)-\left(z_{1}-1\right) z_{13} z_{19}\left(z_{31}-1\right)=0 \text {, } \\
& f_{26}:=\left(z_{1}-1\right) z_{19}\left(z_{20}-1\right)-\left(z_{2}-1\right) z_{6}\left(z_{19}-1\right) z_{20} z_{24}=0 \text {, } \\
& f_{27}:=\left(z_{8}-1\right)\left(z_{18}-1\right)-z_{8} z_{18}\left(z_{26}-1\right)\left(z_{36}-1\right)=0 \text {, } \\
& f_{28}:=\left(z_{11}-1\right)\left(z_{28}-1\right) z_{29}-z_{9}\left(z_{10}-1\right) z_{27} z_{28}\left(z_{29}-1\right)=0 \text {, } \\
& f_{29}:=\left(z_{16}-1\right) z_{17}\left(z_{29}-1\right) z_{35}-\left(z_{11}-1\right) z_{16} z_{29}\left(z_{34}-1\right)=0 \text {, } \\
& f_{30}:=z_{14} z_{15} z_{16} z_{32} z_{33} z_{34}-1=0 \text {, } \\
& f_{31}:=z_{3}\left(z_{20}-1\right) z_{21}\left(z_{33}-1\right)-\left(z_{2}-1\right)\left(z_{15}-1\right) z_{20} z_{33}=0 \text {, } \\
& f_{32}:=z_{4}\left(z_{5}-1\right)\left(z_{22}-1\right)-z_{3}\left(z_{4}-1\right) z_{5} z_{21}\left(z_{23}-1\right)=0 \text {, } \\
& f_{33}:=\left(z_{4}-1\right)\left(z_{15}-1\right)-z_{4} z_{15}\left(z_{22}-1\right)\left(z_{33}-1\right)=0 \text {, } \\
& f_{34}:=\left(z_{7}-1\right) z_{9} z_{27}\left(z_{34}-1\right)-z_{7}\left(z_{16}-1\right)\left(z_{25}-1\right) z_{34}=0 \text {, } \\
& f_{35}:=\left(z_{26}-1\right)\left(z_{30}-1\right)+\left(z_{12}-1\right)\left(z_{25}-1\right) z_{30}=0 \text {. }
\end{aligned}
$$

From the definitions of $f$,'s, for any $z$ in a certain open neighborhood $U$ of the original solution $\boldsymbol{z}^{0}$ in $\boldsymbol{C}^{36}, \boldsymbol{z}$ satisfies both the equations $\left(^{*}\right)$ and $u_{1}=1$ if and only if it satisfies the equations $(* * *)$. In this paper we are concerning only the case where $z$ is near $z^{o}$, so it suffices to consider the reduced deformation space $W \cap U=F^{-1}(0, \cdots, 0)$, where

$$
F: U \subset C^{36} \longrightarrow C^{35}
$$


is the function defined by $F=\left(f_{1}, \cdots, f_{34}, f_{35}\right)$. By the standard argument in [8], the rank of the Jacobian matrix of $F$ at $\boldsymbol{z}^{\circ}$ is 35 , i.e. $\operatorname{rank}(J F)_{\mathbf{z}^{0}}=35$. In our case, it can be shown directly that the rank of the $35 \times 35$-matrix obtained from the Jacobian matrix $J F$ at $z^{\circ}$ by excluding the thirty-sixth column is 35 . Actually, we can calculate the inverse matrix of this $35 \times 35$-matrix. (The computation of finding out the inverse matrix was carried out by sweeping out method with the assistance of a Sun 3 computer.) Then by the implicit function theorem, there is some open neighborhood $O$ of $z_{36}^{o}$ on which there exists a holomorphic mapping $\phi$ such that $z_{1}=\phi_{1}\left(z_{36}\right), \cdots, z_{35}=\phi_{35}\left(z_{36}\right)$ where $\phi=\left(\phi_{1}, \cdots, \phi_{35}\right)$. At the same time we can calculate the values $\left.\frac{d \phi_{\alpha}}{d z_{36}}\right|_{z_{36}^{o}}$ and $\left.\frac{d^{2} \phi_{\alpha}}{d z_{36}^{2}}\right|_{z_{36}^{o}}(\alpha=1, \cdots, 35)$. Put $T$ be the complex valued holomorphic function defined on $O$ as follows:

$$
T\left(z_{36}\right):=\tau\left(\phi\left(z_{36}\right), z_{36}\right) .
$$

Differentiate $T$ by $z_{36}$ until the second order using chain rule of composite mapping and put complex values at $z_{36}^{o}$ of the partial differentiations. Then we obtain the numerical result as follows:

$$
\begin{aligned}
& \left.\frac{d T}{d z_{36}}\right|_{z_{36}^{o}}=0 \\
& \left.\frac{d^{2} T}{d z_{36}^{2}}\right|_{z_{36}^{o}}=8+8 \sqrt{2} \neq 0 .
\end{aligned}
$$

(The computations were carried out with the assistance of a Sun 3 computer.) Thus $\tau$ is not constant on $W \cap U$ near $z^{\circ}$.

From the above argument we can give the following statement:

Proposition 3.1. If $\left(p_{2}, q_{2}\right)$ is close to $\infty$ in $\boldsymbol{R}^{2} \cup\{\infty\}$ and not equal to $\infty$, then the value of $\tau$ at this point $\left(p_{2}, q_{2}\right)$ is not the same as the value of $\tau$ at the original solution $\boldsymbol{z}^{\circ}$.

There is the following theorem which has a close relation to the above proposition :

Theorem (M. Kapovich [4], [5]). Let $\Gamma$ be a discrete subgroup of $P S L$ $(2, \boldsymbol{C})$ and $N=\boldsymbol{H}^{3} / \Gamma$ a hyperbolic 3-manifold of finite volume. Suppose that $n \geqq 2$ and $N$ has $n$ cusp ends, corresponding to the maximal parabolic subgroups $\Delta_{1}, \cdots, \Delta_{n}<\Gamma$. Suppose that the group $\Gamma$ is a congruence subgroup of some Bianchi group with at least two cusps. Then the restriction homomorphisms

$$
r_{x}: H^{1}(\Gamma, s l(2, \boldsymbol{C})) \longrightarrow H^{1}\left(\Delta_{x}, \operatorname{sl}(2, \boldsymbol{C})\right) \quad(x=1, \cdots, n)
$$

are onto, where we consider $\operatorname{sl}(2, \boldsymbol{C})$ as a $\Gamma$-module with respect to the adjoint representation.

From this we can assert the following: for any hyperbolic 3-manifold $N=$ 
$\boldsymbol{H}^{3} / \Gamma$ with two torus cusps, where $\Gamma$ is a congruence subgroup of a Bianchi group, the hyperbolic Dehn surgery on one of the toral ends of $N$ induces the move of the flat structure of the other cusp torus of $N$. But in our case, the complete hyperbolic 3-manifold $D M=\boldsymbol{H}^{3} / \pi$ is not arithmetic (we can see it easily from [6] which states $\boldsymbol{Q}\left(\operatorname{tr}\left(\pi^{(2)}\right)\right)=\boldsymbol{Q}\left(z_{1}, \cdots, z_{36}\right)$ where $\pi^{(2)}=\operatorname{gp}\left\{\gamma^{2} ; \gamma \in \pi\right\}$ and $\operatorname{tr}\left(\pi^{(2)}\right)$ is the set of the traces $\operatorname{tr}(\lambda)$ for all $\left.\lambda \in \pi^{(2)}\right)$. Thus the above theorem does not work on our example. In fact, Neumann-Reid [7] showed that the general case of the above theorem where the arithmeticity condition on $\Gamma$ is withdrawn does not hold. In [7], they constructed concrete examples indicating this: hyperbolic 3-manifolds with two torus cusps each of which admits a deformation of the cusp not affecting the other one. Especially, for each $g$, they showed the existence of a hyperbolic 3-manifold with one torus cusp and totally geodesic boundary of genus $g$ such that deformations induced by the hyperbolic Dehn surgery on the toral end leave the boundary surface invariant. In contrast to the rigid case as these examples, we need something hard (but straightforward) calculations in the moving case as our manifold $M$.

\section{Main theorem.}

Let $\boldsymbol{M}_{g}$ be the Riemann moduli space consisting of all isometry classes of connected closed hyperbolic surfaces of genus $g$. In this section we consider the move in $\boldsymbol{M}_{2}$ of the totally geodesic closed surface $\Sigma$ of genus 2 embedded in $D M$ with respect to the hyperbolic Dehn surgery on the toral end $\mathcal{E}_{2}$.

Let $[R]$ be the equivalent class of a closed surface $R$ of genus 2 , where two closed surfaces $R_{1}$ and $R_{2}$ are equivalent if there is an isometry isotopic to the identity between them (i.e. $[R]$ is a point in the Teichmüller space of the closed surface of genus 2).

Our main theorem is :

THEOREM 4.1. Let $M$ be the 3-manifold which has the complete hyperbolic structure with one torus cusp and connected totally geodesic boundary $\Sigma$ of genus 2 as constructed in $\S 1$. Let $M(p, q)$ be the topological 3-manifold obtained by performing the Dehn surgery of type $(p, q)$ on the framed toral end of $M$. If $(p, q)$ is a coprime pair of integers and $|p|+|q|$ is sufficiently large, then $M(p, q)$ has a hyperbolic structure with connected totally geodesic boundary $\Sigma(p, q)$, and $[\Sigma(p, q)]$ is not equal to $[\Sigma]$.

Proof. By Theorem 5.8.2 in chapter 5.8 of Thurston [9], if $\left(p_{\imath}, q_{\imath}\right)(\imath=1,2)$ are coprime pairs of integers and $\left|p_{\imath}\right|+\left|q_{\imath}\right|$ are sufficiently large, then $D M$ $\left(p_{1}, q_{1} ; p_{2}, q_{2}\right)$ has a complete hyperbolic structure with finite volume. Now consider the case of $\left(p_{1}, q_{1}\right)=\left(p_{2}, q_{2}\right)$. We then abbreviate $\left(p_{1}, q_{1}\right)=(p, q)$. There is an involution $K$ of $D M(p, q ; p, q)$ which interchanges the two copies of $M$ as well as the two ends each other and leaves a closed surface of genus 2 invariant. By Mostow's rigidity theorem, $K$ is homotopic to the isometry $\kappa$ which is still an involution. Consider now a surface which is invariant by $\kappa$. It is homotopic 
to the closed surface invariant by $K$ and must be totally geodesic. Let us call this Riemann surface $\Sigma(p, q)$. Each half of $D M(p, q ; p, q)$ cut along $\Sigma(p, q)$ is homeomorphic to $M(p, q)$.

Assume that $[\Sigma]=[\Sigma(p, q)]$. Then we can glue the two hyperbolic 3manifolds $M$ and $M(p, q)$ by the identity map of $\Sigma$. Call the resulting hyperbolic 3 -manifold $X$. On the other hand, consider the hyperbolic 3-manifold $D M(\infty ; p, q)$ obtained by performing the $(p, q)$-hyperbolic Dehn surgery on the toral end $\mathcal{E}_{2}$. The 3-manifold $X$ is homeomorphic to $D M(\infty ; p, q)$, and both $X$ and $D M(\infty ; p, q)$ are the complete hyperbolic 3-manifolds with one torus cusp. Then by Mostow's rigidity theorem, $X$ is isometric to $D M(\infty ; p, q)$. But by Proposition 3.1 , the moduli of the cusp tori of $X$ and $D M(\infty ; p, q)$ are different. Thus we have obtained a contradiction. Therefore $[\Sigma] \neq[\Sigma(p, q)]$.

COROLLARY 4.2. With the same hypotheses as in Theorem 4.1, the modulus of $\Sigma(p, q)$ is different from that of $\Sigma$ and there are infinitely many $(p, q)$ 's such that $\Sigma(p, q)$ 's are mutually different in $\boldsymbol{M}_{2}$.

When $(p, q)$ is near $\infty, \Sigma(p, q)$ is developed to a hyperplane in $\boldsymbol{H}^{3}$ which is very close to the original one on which $\Sigma$ is developed. Then the fundamental domains of $\Sigma$ and $\Sigma(p, q)$ are very close to each other. Thus this corollary is induced by Theorem 4.1, since the modular group acts on the Teichmüller space properly discontinuously.

Acknowledgements. The author would like to express his sincere gratitude to Professor Shigeyuki Morita and Professor Sadayoshi Kojima for their constant encouragement and many useful suggestions. He acknowledges useful communications and correspondence with Professors Michael Kapovich, Walter Neumann, Alan Reid and Teruhiko Soma. He also wish to thank Mr. Yasushi Yamashita for his supporting of the numerical calculations in $\S 3$.

\section{REFERENCES}

[1] R. BRooks, Circle packings and co-compact extensions of Kleınian groups. Invent. Math. 86 (1986), 461-469.

[2] D.B.A. Epstein And R.C. Penner, Euclidean decompositions of noncompact hyperbolic manifolds. J. Differ. Geom. 27 (1988), 67-80.

[3] M. FuJII, Hyperbolic 3-manifolds with totally geodesic boundary. Osaka J. Math. 27 (1990), 539-553.

[4] M. Kapovich, On faithful representations of fundamental groups of 3-manifolds with toroidal boundary. (Preprint 1990).

[5] M. KAPOVICH, Eisenstein series and Dehn surgery. (Preprint 1991).

[6] W.D. Neumann And A.W. Reid, Arthmetic of hyperbolic manifolds. (Preprint 1990).

[7] W.D. Neumann and A.W. Reid, Rigidity of cusps in deformations of hyperbolic 3-orbifolds. (Preprint 1991). 
[8] W.D. Neumann and D. Zagier, Volumes of hyperbolic three-manifolds. Topology 24 (1985), 307-332.

[9] W.P. Thurston, The Geometry and Topology of 3-Manifolds. Lecture Notes, Princeton: Princeton University Press 1978/79.

Department of Mathematics

TOKYo Institute of TECHNOLOGY

Oh-okayama, Meguro-ku, TOKyo, 152

JAPAN 